\title{
mHealth and Psycho-Physical Well-Being
}

\author{
Jan Derboven \\ Roos Voorend \\ Meaningful Interactions Lab \\ KU Leuven-imec, Belgium \\ Sebastiano Nucera \\ Department of Cognitive Science, \\ Psychology, Educational and \\ Cultural Studies \\ firstname.lastname@kuleuven.be University of Messina, Italy \\ Sebastiano.nucera@unime.it

\section{Gennaro Tartarisco} \\ Flavia Marino \\ Giovanni Pioggia \\ Katleen Van der Gucht \\ National Research Council of ItalyEducational Sciences \\ Institute of Applied Science and KU Leuven, Belgium \\ Intelligent Systems \\ katleen.vandergucht@kuleuven.be \\ Messina, Italy \\ firstname.lastname@cnr.it \\ Permission to make digital or hard copies of all or part of this work for \\ personal or classroom use is granted without fee provided that copies are \\ not made or distributed for profit or commercial advantage and that \\ copies bear this notice and the full citation on the first page. Copyrights \\ for components of this work owned by others than the author(s) must be \\ honored. Abstracting with credit is permitted. To copy otherwise, or \\ republish, to post on servers or to redistribute to lists, requires prior \\ republish, to post on servers or to redistribute to lists, requires
specific permission and/or a fee. Request permissions from \\ specific permission and \\ Permissions@acm.org. \\ NordiCHI'18, September 29-October 3, 2018, Oslo, Norway
c) 2018 Copyright is held by the owner/author(s). Publication rights \\ icensed to ACM. \\ ACM ISBN 978-1-4503-6437-9/18/09...\$15.00 \\ https://doi.org/10.1145/3240167.3240261
}

\begin{abstract}
Both in academia and in industry, mHealth applications have started to address mental health, as well as physical health. Apart from monitoring physical parameters to help patients to manage their conditions themselves, an increasing number of mHealth apps include dedicated sections for mental health based on cognitive behavioural approaches and mindfulness. Nevertheless, the combination of mental health support with specific self-management paradigms poses particular challenges for the design of the technology. While health monitoring apps should efficiently provide information and encourage users to achieve goals, constant access to detailed health data can be overwhelming and induce additional stress and anxiety. This workshop zooms in on mHealth technology that integrates these mental well-being concerns. Specifically, the focus is on design challenges related to the combination of self-management of conditions on the one hand and mental well-being and mindfulness on the other.
\end{abstract}

\section{Author Keywords}

mHealth; self-management; well-being; healthcare; user experience 


\section{ACM Classification Keywords}

H.5.m. Information interfaces and presentation (e.g., HCI): Miscellaneous

\section{Background: mHealth and Well-Being}

The growing world of mHealth apps has opened new possibilities to improve healthcare delivery and clinical outcomes [9]. The use of mobile technologies allows for more effective control and monitoring of physical health parameters and results. Monitoring patients holds the promise of improving treatment compliance, early detection of medical complications and ultimately preventing unnecessary hospitalizations [5].

Furthermore, these technologies can play an important role in patient empowerment, allowing patients to actively engage in self-management of their condition [9].

Apart from physical health management, mental wellbeing is also increasingly being addressed with technology. For instance, in coping with chronic illness and the complications associated with them, it has been shown that health monitoring and prognosis can significantly be improved with cognitive behavioural approaches (CBT) [12]. Combining mobile technologies with CBT for chronic patients can help those seeking treatment to improve their ability to cope with conditions [6]. Literature also suggests that mindfulness and other positive behavioural changes can be seen in patients that get mindfulness-based cognitive therapy (MBCT) [1]. The ability for CBT to address ongoing psychological aspects of the patient experience provides numerous benefits [3]. Positively affected areas include self-monitoring, physical activity, medication and nutrition [6]: these treatment paradigms have relieved people from anxiety, depression and pain.

Well-being is increasingly being addressed in the $\mathrm{HCI}$ (human-computer interaction) community. In the past, several workshops have been organized on the topic. For instance, the CHI 2012 workshop on "Interaction Design and Emotional Wellbeing" [2] and the "Mobile wellbeing" workshop organized at NordiCHI 2016 [7] explored the design challenge of supporting and improving mental well-being through the design of (mobile) technology. More specifically, the "Mobile Wellness" workshop at MobileHCI 2011 [10] also addressed the relationship between health and wellbeing, focusing on the ways in which our mobile devices can aggregate and visualize personal health or well-being data and how these data streams can be presented to encourage interaction, increased awareness and positive behaviour change. This workshop builds on this prior work, specifically targeting the design challenges inherent in the combination of mHealth and psycho-physical well-being approaches.

\section{Challenges in the Combination of mHealth and Psycho-Physical Well-Being}

While mobile technology can be helpful in reaching both physical and mental health goals, the combination of self-management paradigms with mental support poses particular challenges for the design of the technology. For instance, one prominent challenge is aligning the goals of improving well-being and monitoring health data. While mobile phones can be empowering, constant access to detailed health data and information can also distract users from the present moment in undesirable ways [7]. Access to personal health data 
can be overwhelming and confronting [11], while wellbeing aims to reduce such experiences by "attentively living in the moment" [13]. As Zhu et al. suggest, the "goal achievement, instrumental tool use and overly performed regimentation" that mHealth applications often facilitate, "could well all be hinders for mindfulness" [13].

In this alignment of health and well-being goals, a central design decision is choosing the appropriate design approach from a wide range of existing approaches to well-being technology. Zhu et al. have categorized what they call "digital mindfulness applications" in four groups [13]. These groups range from basic "digitalized mindfulness" in which textual, audio or video guidance is offered, to advanced "quantified mindfulness" involving sensors and realtime feedback. In this range of approaches, it has become an important challenge to design and select the appropriate approach in balancing health monitoring with a well-being approach.

Further challenges related to the combination of mHealth and psycho-physical well-being include the possibility to improve the patient's motivation [4] and promote the feeling of being able to follow medical prescriptions, increasing compliance and subjectivity learning gradually new everyday habits [8]. In particular, integrated psycho-physical support is a promising approach to enhance the patients' awareness of their condition and help them disassociate (unhealthy) emotional and behavioural responses from physical sensations and thoughts. This can allow them to better follow health advice provided by the clinicians.

\section{Goals of the Workshop}

In this workshop, we aim to explore how to take advantage of the combination of self-management mHealth functionality and mental health support to enhance patients' resilience and ability to cope with their condition. Exploring the design space of mHealth for psycho-physical well-being, we will address issues related to user research, design and evaluation of mHealth applications that integrate well-being aspects:

- How can we better understand users' motivations and needs for mental support via mHealth in order to develop better mHealth applications? What are appropriate methodologies to understand users' needs for mental support?

- What does the design space for mobile health applications look like, combining practical selfmanagement and support for mental well-being? What are the specific design trade-offs to be made?

- How can mental support in mHealth applications empower patients and mediate change of unhealthy behaviours correlated with chronic conditions? How can we evaluate these effects of mental support?

\section{Workshop Structure}

Participants will be welcomed and introduced to the workshop goals. In the first session, position papers will briefly be presented. As participants will receive copies of accepted papers in advance, each paper will be limited to 10 minutes including Q\&A.

Discussing design trade-offs and challenges based on the introductory presentations will set the scene for the afternoon, in which smaller groups of participants zoom in on specific challenges, using co-creation activities that encourage collaboration and problem solving. 


\section{Planned Outcomes}

The outcomes of the workshop will be available in different formats. First, the workshop proceedings will be available on the workshop website before and after the conference. Second, common themes, trade-offs and challenges discussed during the workshop will be summed up in hand drawn posters and application concepts. If possible, these outcomes will be shared on the workshop website. Third, we plan a joint position paper with the workshop participants in an HCI journal following the workshop.

\section{Acknowledgements}

This workshop is organized based on PRE-LIFE, an FWO-TBM project (grant No T005417N), and HeartMan, a European H2020 project (grant agreement No 689660).

\section{References}

1. M. Allen, A. Bromley, W. Kuyken, and S. J. Sonnenberg. 2009. Participants' experiences of mindfulness-based cognitive therapy:"it changed me in just about every way possible". Behav Cogn Psychother, 37, 4: 413-430.

2. David Coyle, Conor Linehan, Karen Tang, and Sian Lindley. 2012. Interaction design and emotional wellbeing. In CHI '12 Extended Abstracts on Human Factors in Computing Systems (CHI EA '12), 27752778.

3. R. L. Dekker. 2008. Cognitive behavioral therapy for depression in patients with heart failure: a critical review. Nursing Clinics, 43, 1: 155-170.

4. Y.-S. Lee, W.-M. Jung, H. Jang, S. Kim, S.-Y. Chung, and Y. Chae. 2017. The dynamic relationship between emotional and physical states: an observational study of personal health records. Neuropsychiatr Dis Treat, 13: 411.
5. T. McCurdie, S. Tavena, M. Casselman, M. Yeung C. McDaniel, W. Ho, J. Cafazzo. 2012. mHealth consumer apps: the case for user-centered design. Biomedl Instrum Technol, 46, s2: 49-56.

6. F. Norlund, E.M. Olsson, R. Pingel, C. Held, K. Svärdsudd, M. Gulliksson, G. Burell. 2017. Psychological mediators related to clinical outcome in cognitive behavioural therapy for coronary heart disease: A sub-analysis from the SUPRIM trial Eur $J$ Prev Cardiol, 24, 9: 917-925.

7. Mattias Rost, John Rooksby, Alexandra Weilenmann, Thomas Hillman, Pål Dobrin, and Juan Ye. 2016. Mobile Wellbeing. In Proc. NordiCHI '16. Article 137, 3 pages.

8. A. Pantelopoulos and N. G. Bourbakis. 2010. A survey on wearable sensor-based systems for health monitoring and prognosis. IEEE Trans Syst Man Cybern Syst, 40, 1: 1-12.

9. T. J. J. Skinstad and B. A. Farshchian. 2016 Empowerment or concealed compliance?: A review of literature on mobile ICT solutions for patient empowerment. In Proc. PETRA 2016, 54.

10. Konrad Tollmar, Frank Bentley, John Moore, and Alex Olwal. 2011. Mobile wellness: collecting, visualizing and interacting with personal health data. In Proc. MobileHCI '11, 761-763.

11. Bert Vandenberghe, Jasper Vanhoof, Roos Voorend, David Geerts and Fabienne Dobbels. 2018. The 'Self' as Barrier for Self-Management Technologies in Healthcare? In Proc. PervasiveHealth 2018.

12. B. Whalley et al. (2011) Psychological interventions for coronary heart disease. Cochrane Database Syst Rev 2011.

13. Bin Zhu, Anders Hedman, and Haibo Li. 2016. Design digital mindfulness for personal wellbeing. In Proc. OzCHI '16, 626-627. 